\title{
Psicooncología
}

ISSN: 1696-7240

http://dx.doi.org/10.5209/PSIC.59183

\section{Arteterapia familiar en oncología pediátrica}

\author{
Estibaliz Gutierrez Ajamil ${ }^{1}$
}

Recibido: 5 de enero de 2018 / Aceptado: 23 de febrero de 2018

Resumen. El cáncer infantil tiene efectos emocionales no solo en el niño enfermo, sino en todo el sistema familiar. En este estudio se exponen los resultados de una investigación sobre arteterapia con familias en fase de vuelta a casa tras el ingreso en oncología pediátrica. Objetivo: El objetivo de investigación principal consiste en comprender la dinámica familiar durante el proceso creativo para poder intervenir en la restructuración familiar mediante un programa de arteterapia. Con este estudio se busca desarrollar una metodología de trabajo específica con la que ofrecer un acompañamiento terapéutico a las familias en la fase de vuelta a casa. Método: Durante tres años se ha llevado a cabo un programa de arteterapia en el que han participado 10 familias (36 personas). Los datos obtenidos del trabajo de campo se han utilizado para posteriormente poder realizar un análisis cualitativo e inferir una fundamentación teórica al respecto (Grounded Theory). Resultados: Este estudio muestra que el trabajo arteterapéutico conjunto a lo largo de los meses ayuda a los miembros a explorar maneras alternativas de relacionarse, a adaptar sus roles a la realidad que están viviendo y a utilizar el lenguaje simbólico para expresar conceptos y emociones para los que habitualmente las palabras resultan insuficientes. Conclusiones: El programa arteterapéutico en la fase de vuelta a casa permite incidir en algunos problemas propios del proceso de readaptación. Ampliar el foco de intervención al sistema familiar ayuda a los miembros a compartir sus experiencias y crecimiento personal tras el impacto del cáncer infantil.

Palabras clave: Arteterapia; cancer infantil; familia; fase de vuelta a casa; expresión emocional.

\section{[en] Family art therapy in pediatric oncology}

Abstract. Childhood cancer diagnosis has emotional effects not only on the child with cancer, but also on the family system. This study presents the results of a research project about art therapy with families of children with cancer after discharge from a pediatric ward. Objectives: The main objective of the research project is to understand the family dynamics during the creative process. The aim is to develop a specific methodology to offer a therapeutic support to the families when they return home. Method: Over three years 10 families (36 people) have taken part in the art therapy study. After the therapeutic program finalized, the results were analyzed using a qualitative methodology and relate with the Grounded Theory. Results: This studio showed that the overall effect of the family art therapeutic intervention during this period was helpful allowing family members to explore alternative ways of relating, to adapt their roles to the situation they are living and to use symbolic language to express ideas and emotion when words are not enough. Conclusions: The art therapeutic program on the return home after a pediatric ward admission helps the family to face some problems related to the readaptation process. Broaden the therapeutic approach to the family system helped the family members to share their experiences and personal growth after the impact of the childhood cancer.

Keywords: Art Therapy; childhood cancer; family; homecoming phase; emotional expression.

1 Instituto MAP (Música, Arte y Proceso) - Centro Terapéutico. Vitoria-Gasteiz, Álava.

Email: estibaliz@agruparte.com

* Dirección de correspondencia: Instituto MAP (Música, Arte y Proceso). Paseo de la Zumaquera 35, bajo.01006 Vitoria-Gasteiz, Álava. Email: estibaliz@agruparte.com 
Sumario. 1. Introducción 2. Repercusión del cáncer infantil en los miembros de la familia 3. Crisis, adaptación y asistencia familiar 4. Arteterapia en oncología pediátrica 5. Aportaciones del arteterapia familiar en el cáncer infantil 6. Limitaciones de la investigación 7. Conclusiones 8. Referencias bibliográficas.

Cómo citar: Gutierrez Ajamil E. Arteterapia familiar en oncología pediátrica. Psicooncología 2018;15:133-151. Doi: 10.5209/PSIC.59183.

\section{Introducción}

El diagnóstico del cáncer infantil provoca un impacto en todo el sistema familiar y sus consecuencias perduran aun cuando el tratamiento hospitalario finaliza. El ingreso del niño ${ }^{2}$ enfermo en una unidad de oncología pediátrica genera una crisis en el núcleo familiar, desestabilizando la dinámica con la que los miembros habían funcionado hasta el momento. Los padres de los niños con cáncer cumplen permanentemente un rol de cuidadores durante el ingreso, teniendo que contener sus propias emociones para ello. Además, en el caso de tener más de un hijo, los padres se ven obligados a pedir ayuda para atender a los hermanos sanos, lo que les provoca un gran sentimiento de culpabilidad, ya que lo viven como un abandono de sus funciones parentales. Los hermanos de los niños con cáncer también deben aprender a convivir con los efectos de la enfermedad al tener que separarse de su familia durante un tiempo prolongado. Por lo tanto, en la fase de vuelta a casa, las relaciones entre los miembros se verán afectadas por las diferentes experiencias que cada persona ha vivido durante la hospitalización.

Ampliar la mirada sobre el impacto del cáncer más allá del niño enfermo ayuda a tener una perspectiva sistémica del funcionamiento familiar durante el tratamiento. Afortunadamente, hoy en día contamos con diversos estudios que han permitido conocer en profundidad los síntomas que experimentan cada uno de los miembros en función de su rol respecto al niño enfermo (padre, madre, hermano... ${ }^{(1-5)}$. Del mismo modo, a lo largo de las últimas décadas se ha ido especializando la asistencia psicosocial para las familias de niños con cáncer, por lo que hoy en día las unidades de oncología pediátrica y las asociaciones de padres y madres de niños con cáncer están conformadas por equipos multidisciplinares específicos de este ámbito ${ }^{(6)}$.

A continuación se realiza una breve aproximación a la realidad que vive cada uno de los miembros de las familias de niños con cáncer durante el tratamiento, así como el proceso de crisis y adaptación familiar a la que deben hacer frente en la fase de vuelta a casa. Centrándonos el acompañamiento psicosocial en esta etapa de la enfermedad, se exponen algunos de los resultados de la investigación doctoral Arteterapia con Familias de Niños con Cáncer. Dinámica Familiar en la Creación Plástica defendida en 2017, que se ha realizado en colaboración con ASPANAFOA (Asociación de Padres y Madres de Niños con Cáncer de Álava) y la Universidad del País Vasco/EHU. Este estudio parte del análisis cualitativo de un programa de intervención llevado a cabo con 10 familias entre 2013 y 2016, tratando de comprender cómo el lenguaje visual y el proceso creativo compartido por los miembros pueden ayudar en la reorganización y en la normalización de la rutina familiar tras el ingreso hospitalario.

2 Con la intención de evitar un lenguaje reiterativo, en este trabajo se utiliza la palabra niños para hacer referencia a niños y niñas, al igual que padres para hablar de padres y madres y hermanos para incluir a los hermanos y hermanas. 


\section{Repercusión del cáncer infantil en los miembros de la familia}

Teniendo en cuenta que el tratamiento del cáncer en un niño afecta a todo el sistema familiar, cada uno de los miembros debe hacer frente a algunas problemáticas concretas en función de su rol respecto a la enfermedad. En primer lugar, cuando un niño es ingresado en una unidad de oncología pediátrica, la principal limitación que vive es la falta de autonomía, que en los casos de los niños pequeños se ve interrumpida en pleno desarrollo, y en el caso de los adolescentes supone una de las experiencias más frustrantes, ya que estos deben aceptar una pérdida de control sobre su vida actual y su intimidad, volviendo a depender de sus padres ${ }^{(7)}$. La comprensión de la enfermedad, así como la evolución emocional y conductual del niño dependerán de su edad, de la información recibida y de la seguridad o tensión que perciba en el ambiente. Guijarro ${ }^{(2)}$ señala que aunque el hospital es un espacio generador de trastornos infantiles, cada vez más autores consideran estos trastornos como reacciones adaptativas que se dan hasta que el niño oncológico se familiariza con el entorno hospitalario y es capaz de expresar sus miedos, dudas y necesidades emocionales.

Otra dificultad a la que el niño enfermo debe hacer frente son los cambios en la imagen corporal que provoca el tratamiento oncológico, como pueden ser las alteraciones de peso, erupciones cutáneas, cicatrices, pérdida de órganos y extremidades y alopecia. Como consecuencia, $\mathrm{Grau}^{(8)}$ observa que los niños enfermos muestran un autoconcepto más negativo que los niños sanos, percibiéndose a sí mismos diferentes e inferiores. Lizasoáin ${ }^{(9)}$ añade que estos niños tienen una imagen distorsionada de sí mismos, alto nivel de autocrítica, desconfianza en sí mismos y pueden desarrollar una tendencia al aislamiento. Aunque el tratamiento finalice y el paciente pueda volver a casa, los niños con cáncer deben aprender a convivir con las secuelas, tanto físicas como psicológicas. Dado que en las últimas décadas la tasa de supervivencia del cáncer infantil está aumentando visiblemente, una de las necesidades actuales consiste en estudiar las consecuencias del cáncer y su tratamiento a largo plazo, para así poder asegurar a estos pacientes una vida adulta lo más normalizada posible.

En cuanto a los padres y madres, el diagnóstico del cáncer de un hijo genera un impacto tan doloroso que muchas veces resulta traumático, ya que quiebra las expectativas que tenían sobre el futuro y el crecimiento del niño ${ }^{(10)}$. Una vez que los padres son capaces de asimilar la noticia de la enfermedad, la sensación predominante con la que deben a aprender a convivir es la incertidumbre, lo que se denomina "Síndrome de Damocles". La imposibilidad de saber qué puede ocurrir y si el niño sobrevivirá no sólo se da durante el tratamiento oncológico, sino una vez que la enfermedad remite, ya que no se puede descartar la posibilidad de sufrir una recidiva.

Respecto a los hermanos de niños con cáncer no se han comenzado a realizar estudios sobre el impacto de la enfermedad hasta hace relativamente pocos años. Por ello actualmente en muchas publicaciones se hace referencia a ellos como "los grandes olvidados" o "forgotten children". A pesar de no contar con demasiadas investigaciones sobre la realidad de estos niños y adolescentes, es indudable que su estabilidad y su rutina se ven trastocadas para adaptarse a la nueva situación familiar. El principal problema al que se enfrentan es la ausencia de sus padres, porque estos deben trasladarse al hospital para cuidar del niño enfermo. Al observar 
la implicación de los adultos en el hospital, es habitual que los hermanos sientan celos del niño oncológico, lo que posteriormente les acarrea una sensación de culpa ${ }^{(7)}$. También es habitual que los hermanos manifiesten su situación emocional (miedo, tristeza, preocupación, ansiedad, abandono, celos y culpa) a través de problemas de comportamiento. Otra de las dificultades a las que se enfrentan los hermanos es el desconocimiento sobre el cáncer, ya que en ocasiones, con el objetivo de protegerles, la familia les ofrece explicaciones incompletas o alejadas de la realidad, lo que provoca una percepción confusa y distorsionada del proceso oncológico y de lo que está ocurriendo en el hospital ${ }^{(11)}$.

\section{Crisis, adaptación y asistencia familiar}

Desde esta visión global, entendemos que "el cáncer infantil irrumpe en la vida de familias, con todo su bagaje vital, psicológico y social"(12). Una vez que se confirma el diagnóstico del niño las necesidades de la familia cambian, por lo que en la fase de tratamiento es inevitable que se produzca una reorganización del sistema familiar: "la enfermedad asigna nuevos roles y cada familia forma su propia rutina asignando los papeles que sus miembros van a desempeñar. A veces se hace prematuramente fruto de la urgencia del momento y de la necesidad"(10). La vivencia del proceso oncológico será diferente en cada caso, ya que la capacidad para afrontar el cáncer infantil dependerá de los recursos adaptativos que muestre cada familia en situaciones de crisis, así como la fase en la que se encuentre en el momento del diagnóstico haciendo referencia al ciclo vital y a la estructura familiar ${ }^{(13)}$.

Las responsabilidades durante la enfermedad generan nuevas prioridades en los padres, por lo que habitualmente la atención que antes prestaban a la relación conyugal queda relegada para centrar sus esfuerzos en el cuidado de los hijos. A lo largo del tratamiento oncológico los padres pueden experimentar una serie de dificultades como la no equidad de las funciones parentales, el cansancio sobrevenido por el cuidado del niño enfermo, estilos comunicativos y mecanismos de afrontamiento diferentes entre ambos, la sensación continua de angustia e incertidumbre... que a menudo desencadenan conflictos y distanciamientos en la pareja. $\mathrm{Grau}^{(8)}$ afirma que "aunque no existe ninguna evidencia de que el cáncer infantil sea la causa de las rupturas conyugales que en ocasiones se dan en estas familias, puede facilitar la separación al aumentar la tensión o discordia matrimonial que existía antes del diagnóstico, ya que la enfermedad de un hijo supone un reto incluso en los matrimonios más estables".

Para los hermanos de los niños con cáncer la relación con los otros miembros también se complica, sobre todo por la separación del núcleo familiar que viven al quedarse al cuidado de los abuelos o de otros familiares. Además, los hermanos muchas veces no cuentan con espacios en los que poder hablar con un adulto sobre sus miedos y dudas, y para ellos resulta más complicado aprender a expresar sus emociones que para los niños oncológicos, que están cubiertos por un equipo de profesionales preparados para ello. La AECC (Acción Española Contra el Cáncer) llevó a cabo entre 2006 y 2009 un estudio para conocer la capacidad de expresión emocional de los hermanos de niños con cáncer y la comunicación familiar acerca de la enfermedad. Los resultados mostraron que los padres creen que el grado de comunicación con sus hijos es más alto que lo que indica la realidad, ya que durante el tratamiento oncológico estos no conocen las necesidades del hijo sano. También 
observan que cuando las familias finalizan el tratamiento, los padres en un intento de "normalizar" la situación familiar, utilizan un mecanismo de defensa evitativo, tratando de no hablar de temas relativos a la enfermedad ${ }^{(11)}$.

$\mathrm{La}$ "fase de vuelta a casa" tras el ingreso hospitalario supone una reunificación familiar en la que los miembros deben hacer frente a unas dificultades diferentes a las vividas durante el ingreso, como la readaptación del niño enfermo en el entorno escolar, la vuelta a la rutina laboral de los padres y la preocupación por la situación económica de la familia, que suele empeorar durante el tratamiento(7). La remisión del cáncer es un proceso largo en el que la familia debe aprender a convivir con las consecuencias de la enfermedad, teniendo que aceptar las limitaciones físicas causadas por las secuelas de los tratamientos y los posibles síntomas emocionales. Además, la retirada de la asistencia médica y psicológica puede crear una sensación de indefensión y cierto miedo en estas familias. Durante el ingreso los miembros cuentan con una red profesional que les acompaña en los problemas sobrevenidos por el cáncer, y en esta fase deberán utilizar sus propios recursos para ello.

López-Ibor indica que "la asistencia psicológica para estas familias debe adaptarse a todas las fases de la enfermedad, desde el diagnóstico, hospitalización y vuelta a casa, hasta la supervivencia a largo plazo, o las recaídas, muerte y duelo"(12). Gracias a los estudios que se van realizando al respecto, la asistencia psicosocial también se ha ido profesionalizando en las diferentes fases de la enfermedad. Domínguez apunta: "en épocas anteriores se creía que la mejor manera de adaptarse, tras la enfermedad de un hijo, era volver lo antes posible a las rutinas cotidianas que tenía la familia antes del acontecimiento. Ahora sabemos que las familias necesitan redefinir su sentido de la "normalidad" y reconocer que no todo será igual que antes, para evitar los sentimientos de frustración y expectativas no cumplidas al compararse con otras familias que no han sufrido la experiencia de enfermedad de alguno de sus hijos"(14).

En esta línea, cada vez son más los autores que coinciden en la necesidad de realizar una asistencia dirigida a la familia para poder acompañar las crisis, retos y dificultades derivados del cáncer infantil a nivel relacional. Grau, explica: "cuando un niño cae enfermo, toda la familia enferma con él, por lo que los tratamientos deben ser integrales y referidos a todo el núcleo familiar"(15). Lanzarote y Torrado coinciden: "dentro de la enfermedad y su proceso, como dentro de la terapia familiar, cada miembro va a tener un papel fundamental donde no es posible prescindir de ninguno"(16). Tras su investigación Pitillas añade: "la asistencia para esas familias debe ofrecer un marco de trabajo sanitario donde se ayude a los padres a conocer el mundo interior del niño, sus emociones y necesidades"(3).

Con el fin de conocer la situación real de la asistencia psicosocial que se les ofrece a las familias de niños con cáncer a nivel estatal y como parte de la investigación sobre Arteterapia con Familias de Niños con Cáncer, se ha procedido a realizar una encuesta a 22 asociaciones ${ }^{3}$ de padres de niños con cáncer de todas la comunidades autónomas en 2017. Como resultado, se observa que la gran mayoría de las

ASPANAFOA (Álava), ARGAR (Almería), FUNDACIÓN ENRIQUETA VILLAVECCHIA (Barcelona), ALES (Jaén), ASPANOGI (Guipúzcoa), ANDEX (Andalucía), ASPANOA (Aragón), GALBAN (Asturias), ASPANOB (Baleares), PEQUEÑO VALIENTE (Canarias), AMARA (Cantabria), AFANION (Castilla la Mancha), PYFANO (Castilla y León), AFANOC (Cataluña), AOEx (Extremadura), ASANOG (Galicia), FARO (La Rioja), ASIÓN (Madrid), ADANO (Navarra), AFACMUR (Murcia), ASPANION (C. Valenciana) y ASPANOVAS (Vizcaya). 
asociaciones y hospitales ofrecen apoyo psicológico a las familias durante el ingreso, así como en la fase de vuelta a casa, y una vez superada la enfermedad o en el duelo tras el fallecimiento del niño enfermo.

En cuanto al tipo de terapia ofrecida (individual, grupal, o familiar), todas las asociaciones cuentan con servicios de terapia individual para los miembros y en ocasiones realizan grupos de autoayuda. En ninguna de las asociaciones encuestadas se ofrece específicamente terapia familiar, aunque todas afirman que en el caso de que el equipo profesional lo considere necesario para el proceso de alguno de los miembros, se realizan sesiones psicoterapéuticas con más de un miembro (sesiones de pareja, sesiones con padres e hijos, sesiones familiares) como parte del tratamiento individual. Por tanto, los resultados de la encuesta muestran la necesidad de continuar desarrollando recursos asistenciales que permitan a los profesionales de las unidades y asociaciones de oncología pediátrica realizar intervenciones capaces de recoger las necesidades de todo el núcleo familiar.

\section{Arteterapia en oncología pediátrica}

A lo largo de la historia la expresión artística ha sido utilizada como herramienta para el trabajo con personas que sufren enfermedades físicas y mentales. Como muestra Collette ${ }^{(17)}$, el arteteterapia se ha ido profesionalizando para asistir a diversos colectivos, y hoy en día contamos con estudios que abalan su eficacia para la intervención con pacientes de cáncer. En las unidades de oncología pediátrica cada vez es más común encontrar actividades, propuestas e intervenciones relacionadas con la creación artística, por lo que resulta necesario comprender en qué consiste el arteterapia para poder discernir una intervención terapéutica de un taller artístico dentro de un hospital. Rico matiza la función del arteterapia en oncología pediátrica: "este enfoque va mucho más allá de entretener, que es lo que desde la ignorancia muchas veces se encomienda el arteterapia. Nos enfrentamos a los problemas, hasta el más duro del dolor, pero buscamos una orientación positiva de ello que, desde luego, no es escarbar en el sufrimiento sin más sentido que destapar heridas que no sepamos calmar'"(18).

Como define J.P. Klein, "el arteterapia es una simbolización acompañada"(19) . Para ello, el profesional debe contar con una formación específica en este campo que le permita favorecer y asistir el proceso creativo del paciente, ajustándose a sus ritmos, necesidades y capacidades, de forma que éste sea capaz de ir proyectando su mundo interno en la creación plástica. Durante las sesiones, paciente y arteterapeuta van dando lugar a una vía de expresión alternativa a la palabra, donde el lenguaje simbólico permite comunicar emociones e ideas confusas, contradictorias y dolorosas que en muchas ocasiones no es posible nombrar. Parte de las funciones del arteterapeuta consisten en saber leer ese discurso visual con el que el paciente se muestra mientras crea, ya que la expresión artística le da la posibilidad de manifestarse de una forma ambigua, sutil, o metafórica que respete sus mecanismos de defensa ante la realidad que está viviendo.

En el caso de arteterapia en oncología pediátrica resulta imprescindible comprender el significado que la enfermedad tiene para el paciente, porque como apunta López-Ibor, no hay que olvidar que "el cáncer es un hecho circunstancial en la vida del niño, que continua su desarrollo evolutivo y madurativo"(12). Bengoechea 
y Moreira ${ }^{(20)}$ reflexionan sobre el potencial que las terapias creativas pueden aportar en este contexto atendiendo a la predisposición natural de los niños a crear, jugar y experimentar. Estas autoras observan que el proceso creativo permite que lo que podría ser una experiencia traumática se convierta en una experiencia de refuerzo a través de vivencias motivadoras, ilusionantes y placenteras que se integren dentro del entorno médico.

Las intervenciones realizadas en diferentes unidades pediátricas muestran que el trabajo arteterapéutico aporta a estos niños y adolescentes la posibilidad de vivir situaciones poco frecuentes en la hospitalización, como la toma de decisiones o la expresión emocional ${ }^{(21-23)}$. Los espacios de arteterapia ayudan a los niños enfermos resignificar algunos aspectos de su biografía en un lenguaje adaptado a su edad y situación vital, así como a identificarse con un rol más activo, dejando temporalmente de ser pacientes receptores del tratamiento para poder observarse como agentes activos de su proceso y experiencia.

Respecto al lenguaje simbólico de los niños ingresados en oncología pediátrica, Esquembre y Pérez ${ }^{(24)}$ explican que es frecuente que los pacientes elijan como motivo de sus creaciones batallas y luchas entre personajes fantásticos, superhéroes y figuras con grandes poderes, o propongan juegos en los que eligen roles de personajes sanadores como médicos o veterinarios que buscan curar enfermos, emitir diagnósticos, hablar de síntomas, etc. Estos autores coinciden con otros arteterapeutas del ámbito oncológico que observan cómo muchos de los personajes que crean los pacientes son representaciones metafóricas de un "dañador" y un "reparador", permitiendo que el niño proyecte sus vivencias en forma de juego, y sea capaz de ir asimilando lo ocurrido desde sus recursos y necesidades.

Esquembre y Pérez ${ }^{(24)}$ también identifican otro tipo de obras con una gran carga inconsciente en las que la imagen encarna la contradicción e incertidumbre de los pacientes, como un paisaje en el que se integran una tormenta y el sol. La representación de dichos elementos en las producciones plásticas muestra las contradicciones con las que conviven los niños durante el tratamiento oncológico, ya que deben sufrir procedimientos dolorosos para poder sanar y se plantean dudas sobre la muerte y el futuro que los adultos no saben resolver. Por ese motivo, arteterapia supone para el niño con cáncer un espacio en el que es posible expresar su confusión a través del juego creativo, ser comprendido mediante imágenes simbólicas y sentirse reconocido en su proceso de crecimiento durante el tratamiento hospitalario.

\section{Aportaciones del arteterapia familiar en el cáncer infantil}

Hasta el momento, generalmente las terapias creativas en oncología pediátrica están dirigidas al paciente durante el ingreso, realizando sesiones en formato "cama a cama", donde el arteterapeuta porta una serie de materiales con los que trabajar en las habitaciones de los niños enfermos. En algunos casos, los familiares, cuidadores o personal sanitario que se encuentren ese momento con el niño forman parte de la sesión, como observadores o de una forma más activa.

En la encuesta realizada a varias asociaciones de padres y madres de niños con cáncer también se preguntó por la oferta de terapias creativas en este contexto. Los resultados muestran que 13 de las 22 asociaciones cuentan con terapias creativas: 6 
ofrecen musicoterapia ${ }^{4}, 4$ arteterapia ${ }^{5}$ y 3 ambas modalidades ${ }^{6}$ durante el tratamiento hospitalario de los niños. Una vez que el paciente se encuentra en fase de vuelta a casa, 5 asociaciones ${ }^{7}$ continúan ofertando terapias creativas en el caso de que la familia lo demande. Estas sesiones se hacen con una metodología individual, grupal o en forma de taller con los niños enfermos, pero en la encuesta no se han encontrado programas de arteterapia dirigidos a padres, madres y hermanos de niños con cáncer. Por ese motivo, la investigación sobre Arteterapia con Familias de Niños con Cáncer propone dos perspectivas innovadoras en este campo: la intervención arteterapéutica con todo el núcleo familiar del niño enfermo, y el acompañamiento de estas familias en la fase de vuelta a casa tras el tratamiento hospitalario.

Dada la limitación de publicaciones sobre programas y estudios previos en los que poder basar la intervención ${ }^{(25-29)}$, esta investigación se ha realizado bajo una metodología cualitativa (Grounded Theory ${ }^{(30)}$ ) que busca extraer los datos observados en el trabajo de campo para posteriormente poder inferir una teoría fundamentada generalizable, en este caso, al trabajo arteterapéutico con familias de niños con cáncer en fase de vuelta a casa. Para llevar a cabo la investigación se ha asistido a 10 familias con un niño o adolescente con cáncer en fase de mantenimiento (con consultas externas periódicas), en la que los miembros de la familia estén realizando la reunificación familiar y la vuelta a la rutina escolar o laboral tras el ingreso en la unidad de oncología pediátrica del Hospital de Cruces (Bilbao). En total en el estudio han participado 36 personas: 20 padres y madres, 9 niños/as o adolescentes con cáncer y 7 hermanos/as.

Las sesiones se han realizado en modalidad familiar, es decir, todos los miembros que componen el núcleo familiar han asistido conjuntamente a sesiones semanales de una hora a lo largo de un curso escolar, haciendo una media de 17 sesiones por familia. A lo largo de los meses la arteterapeuta ha ido ofreciendo una serie de materiales o propuestas artísticas con los que los participantes han podido experimentar, compartiendo sus experiencias creativas y resultados plásticos con los miembros de su familia. El trabajo de campo ha sido registrado de forma audiovisual y fotográfica, y supervisado por un equipo de consulta formado por 6 terapeutas de los ámbitos relacionados (arteterapia, psicooncología infantil y terapia familiar). En la fase de interpretación de resultados, se han identificado los datos generalizables a todas las familias, analizándolos y codificándolos para posteriormente estructurar un modelo teórico de intervención basado en la realidad observada en el trabajo arteterapéutico. En esta teoría fundamentada se han conceptualizado una serie de aspectos relevantes a tener en cuenta a la hora de diseñar un programa de intervención en el contexto de la vuelta a casa:

1. La realidad social, cultural, económica, familiar, emocional... de las familias que han participado en el programa de arteterapia.

4 ARGAR (Almería), ASPANOA (Aragón), ASPANOB (Baleares), ADANO (Navarra), ASPANOVAS (Vizcaya) y FUNDACIÓN ENRIQUETA VILLAVECHIA (Barcelona).

ASPANIÓN (Alicante), ANDEX (Andalucía), ASPANOGI (Guipúzcoa) y ASPANAFOA (Álava).

GALBAN (Asturias) y AFANOC (Cataluña) y ASANOG (Galicia).

GALBAN (Asturias), ASPANOA (Aragón), ADANO (Navarra), ASPANOVAS (Vizcaya) y ASPANAFOA (Álava). 
2. El setting: las especificidades del espacio físico de las sesiones para el trabajo con familias y la adaptación de los materiales y disposición de los elementos de trabajo para los niños con cáncer.

3. La temporalización de las sesiones y la duración del programa de intervención en función de los objetivos terapéuticos.

4. El rol y las áreas de intervención del arteterapeuta familiar.

5. El tipo de materiales, técnicas y propuestas plásticas ofrecidas a las familias en función de los objetivos específicos (favorecer la narrativa, el simbolismo, la expresión emocional, la improvisación, el trabajo individual, la comunicación entre los miembros...).

6. Las modalidades de trabajo (individual/ diádico/ conjunto o familiar) para promover a través del proceso de creación unos vínculos alternativos a los vividos durante el ingreso hospitalario.

7. La utilidad del lenguaje visual y de las representaciones simbólicas en la comunicación intrafamiliar tras la experiencia del cáncer infantil.

Además, en este estudio se ha diseñado una Evaluación Familiar Inicial (EFI) y Evaluación Familiar Final (EFF) basada en la observación de la dinámica de los miembros durante el proceso creativo. Para ello se estructuran las dos primeras y las dos últimas sesiones de cada familia con una propuesta de trabajo concreta, y posteriormente se contrasta su respuesta al inicio y al final del proceso arteterapéutico. Tras realizar la comparación de la dinámica familiar en la EFI y la EFF, se han identificado las coincidencias en las familias participantes, observando algunos resultados generalizables que muestran la utilidad del arteterapia familiar en la fase de vuelta a casa:

En primer lugar se ha analizado el proceso creativo individual de los miembros. En las primeras sesiones se observa que menos de la mitad de los adultos realizan una producción individualmente, mientras que la mayoría de padres y madres adoptan un rol de "auxiliares" en el proceso creativo de sus hijos. En las últimas sesiones en cambio, a excepción de 2 personas, todos los participantes realizan su propia obra de forma autónoma, lo que indica un desarrollo de sus habilidades creativas y un aumento de la seguridad en su trabajo personal. En el caso de los padres, esta capacidad de realizar su propia creación plástica se entiende como el resultado de un trabajo continuo de introspección durante el proceso arteterapéutico, logrando disfrutar de la experimentación y pudiendo reconocerse a sí mismos en una actitud más lúdica con la que se permitan improvisar sin juzgarse. En este sentido, las sesiones de arteterapia sirven a los adultos para poder dejar temporalmente de lado su función de cuidadores, lo que genera un ambiente más relajado que les permite acercarse a sus hijos a través del juego compartido.

En el análisis del proceso creativo conjunto también se han encontrado diferencias entre la EFI y la EFF respecto a la dinámica familiar. En concreto, se han observado unas variaciones significativas en cuanto a la toma de decisiones y a la distribución del trabajo. En las primeras sesiones las familias tienden a delegar la toma de decisiones sobre el motivo de la obra y las técnicas a utilizar en uno de los hijos. Como pauta general, ese hijo o hija coordina el proceso creativo mientras que los padres, madres y hermanos colaboran de una forma más periférica. En otros casos, cada miembro trabaja de forma independiente realizando su propia obra, aunque todos los miembros compartan un mismo soporte. En las últimas sesiones en cambio, 
se observa que las familias dedican un tiempo para la toma de decisiones y el reparto del espacio en el soporte. En estas sesiones finales el motivo de la obra suele ser consensuado por todos, combinando las ideas de varios miembros y definiendo en qué parte del soporte va a intervenir cada uno de ellos.

Estas variaciones en la toma de decisiones previa al proceso creativo muestran un desarrollo de la dinámica familiar en la que los miembros ya se reconocen y se complementan entre sí a la hora de realizar un proyecto común. El reparto del espacio sobre el soporte responde a un deseo de la familia de que todos los miembros formen parte del resultado final, aceptando e integrando las habilidades creativas de cada uno. En cuanto al subsistema fraternal se advierte una modificación de los roles respecto a la etapa del ingreso hospitalario: en las sesiones de arteterapia el niño enfermo no es el protagonista ni los hermanos deben quedarse al margen, sino que se genera un mayor equilibrio en la familia, siendo igualmente escuchados y atendidos todos los hijos.

Por último, en el análisis comparativo EFI-EFF también se han constatado diferencias en la elección del motivo o temas representados en las obras. Al contrastar las producciones realizadas de forma conjunta por el núcleo familiar al inicio y al final del programa de arteterapia, se observa que en las primeras sesiones los participantes realizan elementos estereotipados o que ya saben dibujar previamente (personajes, paisajes...). En cambio, en la última obra conjunta, todas las familias excepto una decidieron representarse a sí mismas a modo de autorretrato familiar. Estos autorretratos en algunos casos son figurativos, dibujando(se) cada miembro de una forma intencionadamente realista, y en otros casos son retratos con una carga más simbólica, en la que cada uno de los miembros está representado por su color favorito, por su inicial, etc. Esta reiteración del tema "nuestra familia" o "nosotros" como obra final del proceso arteterapéutico indica dos ideas relevantes en el desarrollo de la autopercepción de las familias: por un lado, muestra la necesidad de redefinirse como sistema tras la experiencia vivida en oncología pediátrica. Por otro lado, la capacidad de realizar autorretratos simbólicos confirma la utilidad del lenguaje metafórico y visual a la hora de expresar ideas o emociones difícilmente explicables con palabras, como en este caso es la adaptación de la dinámica familiar a la nueva realidad que están viviendo.

Ahondando en el lenguaje simbólico de los miembros más allá de las sesiones de EFI y de EFF, también se han constatado algunos elementos recurrentes en todas las familias participantes. Resulta importante señalar que la metodología utilizada en este proyecto ha sido un sistema de trabajo no directivo, lo que implica que la arteterapeuta ofrece materiales y técnicas de trabajo concretas en las sesiones, pero no hace propuestas sobre el tema o motivo de la obra. La metodología no directiva en arteterapia favorece la proyección de aspectos psíquicos de la persona en su obra, permitiendo que, en este caso, cada miembro o familia desarrolle un lenguaje simbólico propio con el que expresarse y comunicarse. Por lo tanto, la reiteración de algunos motivos en las producciones expresa una necesidad de elaborar ciertas ideas o emociones por parte de los participantes en el estudio.

La primera observación respecto a las obras realizadas en el programa de arteterapia es que "la familia" ha sido el motivo de la obra más recurrente en todos los casos, independientemente de que los miembros trabajasen en modalidad individual, diádica o conjunta, y de los materiales o técnicas ofrecidas. No solo se han creado autorretratos familiares como ocurre en las sesiones de EFF, sino que los participantes 
también han realizado familias figurativas de otras tipologías (familias de animales, familias de robots, familias de monstruos...). Otro de los recursos más habituales para autorreferenciarse de forma visual a lo largo de la experiencia de todas las familias ha sido estampar sus huellas con pintura acrílica o marcar el contorno de las manos con técnicas secas, logrando representar así a todos los miembros sobre un mismo soporte, y al mismo tiempo, dejando una huella física y tangible de esa experiencia (véase figura 1).

Al igual que ocurre en las sesiones de arteterapia que se realizan en las unidades de oncología pediátrica, en la fase de vuelta a casa también es frecuente que los niños representen personajes mágicos o con superpoderes, con la capacidad de curar o de dañar. Además, la mayoría de niños con cáncer entre 3 y 6 años que han participado en este estudio en algún momento del programa han utilizado los materiales para crear disfraces con los que convertirse ellos mismos en guerreros, brujas o reinas, haciendo que su propio cuerpo cumpla el papel de soporte de la obra. Este hecho resulta especialmente significativo si se tiene en cuenta que estos niños han vivido un ingreso prolongado en el que el personal sanitario debía revisar diariamente las secuelas físicas en sus cuerpos, y han tenido que someterse a procedimientos dolorosos con la dificultad añadida de tener que ser "valientes, fuertes, y obedientes" durante el tratamiento. En esta fase de vuelta a casa, que los niños decidan decorar o transformar su aspecto a través de la creación plástica muestra una reapropiación corporal, es decir, la capacidad de volver a sentirse dueños de su corporalidad. Integrar su imagen física actual dentro de la obra permite elaborar a través del juego su autopercepción y su autoestima, dos áreas que habitualmente resultan afectadas por la enfermedad.

En el caso de los padres y madres también se ha detectado un lenguaje común en la mayoría de las familias, no tanto en lo referente a los elementos creados, sino al

Figura 1. Detalle de obra conjunta-familiar. Técnicas secas sobre papel

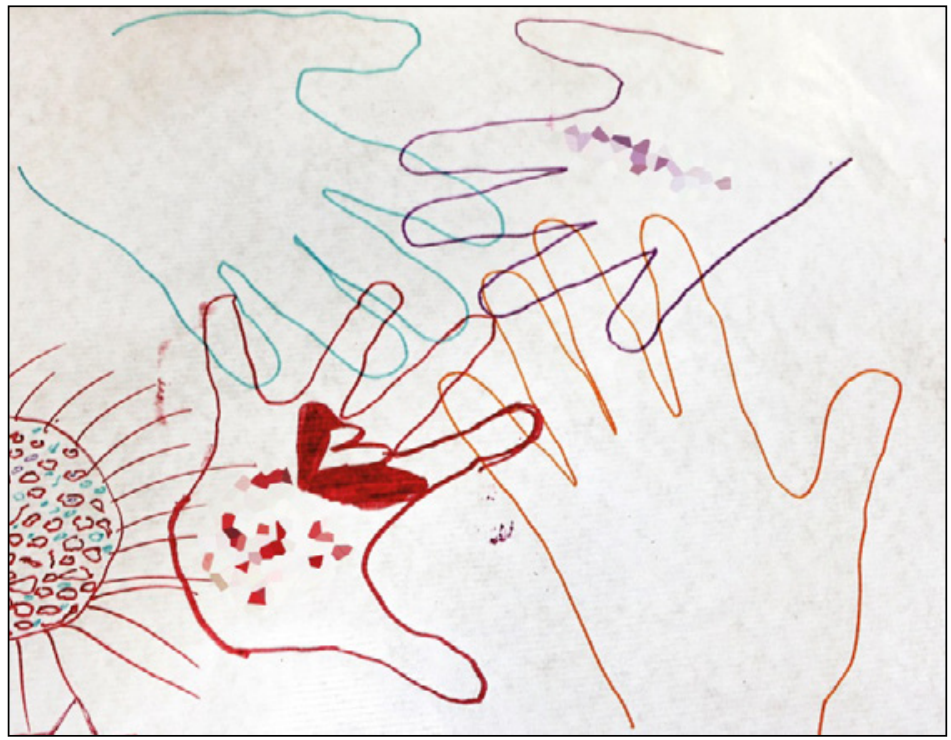


significado que los autores dan a sus obras. En todos los casos se observa que ante las propuestas de creación conjunta o diádica, los niños tienden a hacer personajes, mientras que los padres y las madres crean elementos que sostengan o protejan a dichos personajes (paisajes, casas, vehículos...). Este concepto de "defensa" también se repite en muchas de las obras individuales de los padres y madres, a veces de forma más explícita: "He dibujado un paraguas para que mis hijos puedan protegerse si llueve", y en otros casos proyectado hacia personajes vulnerables: "Esto es un paisaje con cráteres para que los extraterrestres pequeños puedan refugiarse" ${ }^{\text {. El }}$ discurso metafórico generado en las sesiones de arteterapia sirve a los adultos para transmitir mensajes de protección y cuidado hacia sus hijos también a través de las producciones plásticas.

A pesar de que a las familias no se les ha propuesto ningún tema de trabajo a lo largo del programa, los participantes han realizado algunas obras acerca de la enfermedad, aunque en este caso se observan diferencias en función del rol que ha cumplido cada miembro respecto al cáncer. Los niños afectados hacen referencia a las secuelas de la enfermedad desde un discurso predominantemente emocional, como ejemplo, un niño de 6 años con leucemia creó "una bufanda para no volver a ponerme enfermo", y una niña de 5 años con tumor cerebral construyó "una muñeca con pelo y otra sin pelo" 9 . Los padres y madres en cambio, para representar aspectos de su vivencia en oncología, utilizan materiales que permiten un trabajo más cognitivo y añaden elementos verbales a través del collage que les ayuden a realizar un discurso racional sobre su aprendizaje o crecimiento personal a partir del diagnóstico de sus hijos (véase figura 2). Por último, en las obras de los hermanos de niños con cáncer los recursos gráficos son más diversos y no se han detectado elementos comunes entre sí, aunque se observan producciones en las que predomina la confusión y la contradicción. En algunos casos, a través del lenguaje simbólico en las sesiones ha sido posible elaborar con los hermanos la ambivalencia que sienten hacia el niño enfermo, dando lugar a obras donde se superponen emociones de cariño, enfado, admiración, celos, etc.

Con el objetivo de poder comprender los beneficios del arteterapia para trabajar con familias de niños con cáncer, además de llevar a cabo un estudio sistemático del proceso creativo y del lenguaje simbólico de los miembros, también se ha querido conocer la percepción en primera persona de las familias participantes en la investigación. Para ello, tras finalizar las el programa de intervención se ha realizado una encuesta individual de 13 preguntas abiertas a cada participante con la que poder contrastar las tres perspectivas: arteterapeuta-investigadora, profesionales externos y familias asistidas. En dicha encuesta se formula la siguiente pregunta ¿En qué crees que puede ayudar un programa de arteterapia a familias que están realizando la vuelta a casa tras el tratamiento en oncología pediátrica? En el análisis de las respuestas se han identificado 4 grandes conceptos en los que coinciden los participantes, y que confirman algunos de los resultados observados en el estudio: Reunificación familiar/Disfrutar de la creación y el juego/Readaptación de la dinámica familiar / Expresión emocional. A continuación se recogen algunos fragmentos literales de las respuestas recibidas a esta pregunta, utilizando la sigla M. para citar a las madres, P. para los padres, N.O. para los niños oncológicos y H. para los hermanos.

Citas literales de padres de niños con cáncer.

Citas literales de niños con cáncer. 
Figura 2. Autorretrato simbólico. Detalle de una obra de R., madre de una niña con cáncer. Collage y técnicas secas sobre papel.

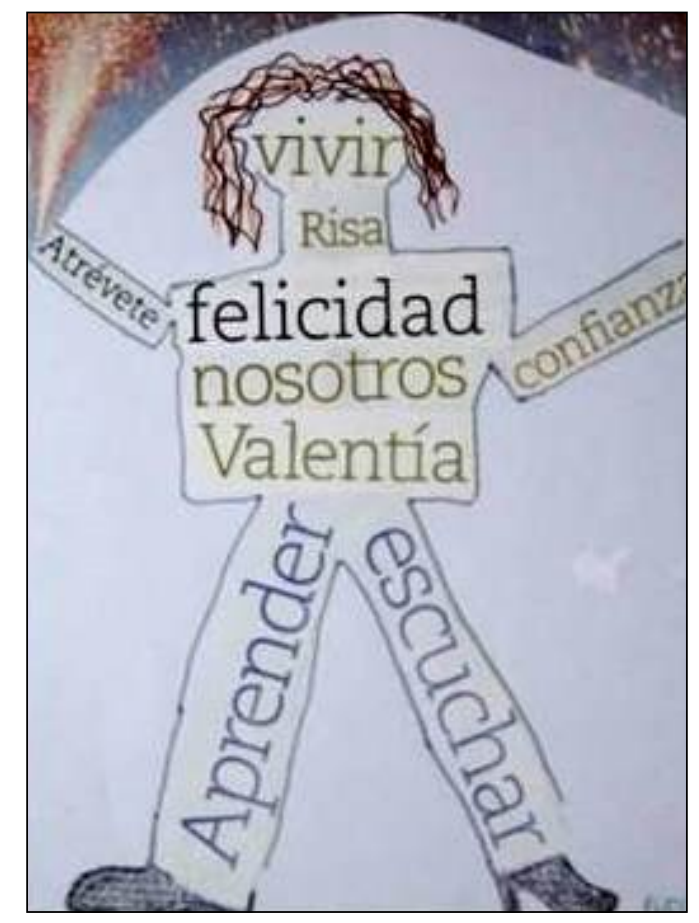

¿En qué crees que puede ayudar un programa de arteterapia a familias que están realizando la vuelta a casa tras el tratamiento en oncología pediátrica?

Concepto recurrente: Reunificación familiar

H (7 años): "Para estar juntos, para pasar un rato juntos y aprovechar".

P: "Después de una muy mala experiencia permite volver a disfrutar de la familia". H (4 años): "Les gustará para volver a estar todos juntos".

H (5 años): "Para estar juntos porque han estado mucho tiempo en el médico".

N.O. (10 años): "Para que vuelvan a estar juntos, estar más unidos, hacer cosas entre todos, que la familia esté junta para siempre".

Concepto recurrente: Disfrutar de la creación y el juego

N.O. (5 años): "Les va a gustar. Porque hacemos lo que queremos: pintamos, hacemos plasti, hacemos mariposas con pintura..."

P: "Para encontrar un espacio y un tiempo donde olvidar un poco los problemas gordos y relajarse y divertirse en familia".

N.O. (16 años): "Despejarse la mente y separarse de todo".

H (5 años): "Aprender a pintar mejor".

N.O. (4 años): "Para que hagan muchos ejercicios. Que vengan las familias a pasárselo bien". 
Concepto recurrente: Readaptación de la dinámica familiar

M: "Cambiar la rutina hospitalaria y concentrarte en otra cosa que no sea la enfermedad, y poder hacer cosas normales".

P: "Para olvidarte de todo y recuperar la familia porque siempre hay alguien abandonado".

M: "Normalizar esa situación que nunca debió ocurrir".

M: "Puede ayudar a recuperar en el caso de tener hermanos sanos, la unión anterior a la aparición de la enfermedad, incluso reforzarla".

M: "Creo que les vendría bien para evadirse por un rato de lo que están viviendo todos los días. Hacer algo diferente juntos, algo divertido, que les haga disfrutar de ellos y acercarse de otra forma".

M: "Es una forma de pensar en otra cosa. Cada vez vemos más alejados los malos momentos de hospitalización y miramos más hacia delante".

P: "Pues ayudarles a reubicarse y empezar a funcionar de nuevo como familia tras una experiencia traumática que puede enrarecer las relaciones".

\section{Concepto recurrente: Expresión emocional}

P: "Considero que nos hemos dicho cosas mediante la plástica. Creo que aporta eso, comunicación no verbal”.

M: "Que alguien puede expresar mucho sobre el papel, en cierto modo descargando ese sentimiento".

H (12 años): "Expresar lo que han sentido. Cosas que te has perdido y las puedes expresar".

N.O. (13 años): "Para desahogarse".

$\mathrm{H}$ (8 años): "A expresarse mejor, a sorprenderse con ellos mismos y a ver que no hace falta ser un artista para hacer cosas increíbles".

M: “Ayuda a ordenar emociones, a sacarlas, a sentir cómo estás y cómo está el niño enfermo y su hermano".

\section{Limitaciones de la investigación}

La investigación sobre Arteterapia con Familias de Niños con Cáncer parte de una detección de necesidades en ASPANAFOA (Asociación de Padres y Madres de Niños con Cáncer de Álava), entidad que en 2013 se plantea ofertar un servicio arteterapéutico específico para el núcleo familiar de los niños con cáncer. Hasta el momento contamos con un número escaso de publicaciones sobre arteterapia familiar, la mayoría de ellos siendo experiencias de trabajo puntuales en espacios de psicoterapia sistémica en EE.UU., en los que el proceso creativo es utilizado como recurso para observar la dinámica familiar en las primeras entrevistas. En el caso de ASPANAFOA, se propone realizar un acompañamiento a las familias a lo largo de la fase de vuelta a casa, utilizando para ello la creación plástica como eje principal todas las sesiones a lo largo de los meses. Tras constatar que no existen servicios similares en otras asociaciones ni unidades de oncología pediátrica, se optó por diseñar un programa de arteterapia para familias de niños con cáncer a modo de investigación doctoral. 
Dado que el objetivo principal consistía en comprender las especificidades del arteterapia familiar en oncología pediátrica, se escogió una metodología cualitativa que permitiese identificar los fenómenos relacionales que se diesen durante el proceso creativo. Los datos obtenidos a lo largo de todo el programa de intervención, incluidos los ítems de la Evaluación Familiar Inicial y la Evaluación Familiar Final se han recogido desde una perspectiva cualitativa, tratando de comprender qué aporta a las familias crear de forma conjunta. Por ese motivo, los resultados de esta investigación suponen un primer acercamiento teórico al arteterapia familiar en oncología pediátrica. Una vez desarrollada la fundamentación respecto al setting, a las técnicas y al rol de arteterapeuta familiar, es posible avanzar en el desarrollo de esta metodología de intervención, profundizando en ella con estudios similares que utilicen sistemas de medición cuantitativos con los que constatar la evolución de los miembros participantes.

La metodología de investigación utilizada (Grounded Theory o Teoría Fundamentada) es útil a la hora de proponer modelos teóricos aplicables a nuevas áreas de estudio, pero también conlleva una serie de limitaciones. La singularidad de esa metodología consiste en identificar, categorizar y conceptualizar datos generalizables, por lo que se deben descartar los fenómenos excepcionales o puntuales que se hayan observado. El uso de la Teoría Fundamentada en este caso ha permitido elaborar una propuesta teórica de intervención para familias de niños con cáncer en fase de vuelta a casa, pero ha sido necesario excluir de la publicación otros datos relevantes que se han detectado en el desarrollo de las sesiones, como el proceso arteterapéutico con adolescentes con cáncer o con las familias de niños que no sobreviven a la enfermedad.

En el trabajo de campo se ha asistido a 10 familias de niños con cáncer que se han prestado voluntariamente a formar parte de una investigación ${ }^{(31)}$, en la que criterio de inclusión era encontrarse en la fase de vuelta a casa tras el tratamiento hospitalario, lo que ha permitido trabajar con múltiples tipologías familiares y estadios de la enfermedad. Teniendo en cuenta que las familias que han vivido la experiencia oncológica con niños o con adolescentes se encuentran en etapas distintas del ciclo vital, se han identificado algunas especificidades en cuanto al trabajo con adolescentes con cáncer. Dichos datos no son generalizables por no contar con un número suficiente de este tipo de familias en la investigación, aunque sí se han identificado algunos rasgos comunes en los adolescentes que han participado en el estudio, como el rechazo a trabajar sobre su álbum fotográfico familiar, la necesidad de rivalizar con sus padres respecto al resultado plástico de las obras y la capacidad de transmitir conceptos abstractos a través del lenguaje simbólico, difiriendo del proceso creativo de los niños menores de 11 años.

Del mismo modo, también se han detectado diferencias en el trabajo arteterapéutico con las familias en función de la situación de la enfermedad, encontrando casos concretos y excepciones en los que los resultados no son generalizables, y por tanto no se han incluido en la fundamentación teórica. Como ejemplo de ello, se ha acompañado la despedida de una familia en el final de la vida del niño enfermo, así como a una familia en la que la niña había fallecido y la asistencia arteterapéutica se centraba en el duelo de los padres y del hermano de 5 años. Estos modelos de familias en fase de vuelta a casa requieren una metodología de intervención específica, ya que la readaptación de la dinámica familiar tras el tratamiento hospitalario implica asumir la pérdida de un hijo o de un hermano. Por 
ese motivo, resulta necesario continuar estudiando las aportaciones del proceso creativo y del lenguaje visual en arteterapia a la hora de abordar la muerte y el duelo en la familia, para poder ofrecer nuevas vías de comunicación y acompañar procesos vitales en los que las palabras no son suficientes.

\section{Conclusiones}

El ingreso en una unidad de oncología pediátrica conlleva una serie de retos y dificultades que desestabilizan la dinámica y la rutina de todos los familiares, afectando directamente a las relaciones entre los miembros. Por ello, en la fase de vuelta a casa se ponen en juego diversas problemáticas afectivas y vinculares derivadas de la experiencia hospitalaria. La reunificación familiar precisa un periodo de adaptación en la que los miembros deben asimilar las secuelas de la enfermedad y aprender a convivir con ciertas limitaciones y pérdidas, además de la incertidumbre y el miedo a la recidiva.

Como se observa en los resultados de la investigación de Arteterapia con Familias de Niños con Cáncer, trabajar con el núcleo familiar en la fase de vuelta a casa permite incidir en algunas de las dificultades específicas que viven los miembros en esta etapa de la enfermedad. Tras una primera fase de observación para identificar las alianzas y coaliciones que se hayan podido establecer entre los miembros durante el ingreso, el acompañamiento arteterapéutico ofrece un espacio en el que trabajar la reagrupación familiar y explorar diferentes roles con los que los usuarios se sientan reconocidos y valorados. El programa de arteterapia ayuda a la familia a realizar su propio proceso de reunificación, atendiendo las necesidades individuales de los miembros, aceptando las secuelas físicas, psíquicas y emocionales del cáncer infantil $\mathrm{y}$ tratando de disminuir los mecanismos defensivos o evitativos en el entorno familiar de los niños y adolescentes afectados.

El proceso terapéutico en la fase de vuelta a casa representa para la familia un periodo de transición entre el ingreso en la unidad de oncología pediátrica y la vuelta a la rutina escolar y laboral. Tras la incertidumbre vivida durante el tratamiento hospitalario, el espacio arteterapéutico en la fase de vuelta a casa debe ofrecer una contención estable y periódica que le permita a la familia recomponerse respetando sus ritmos. El cáncer, observado desde el prisma de una crisis vital, también conlleva un crecimiento personal en el que cada miembro se cuestiona sus necesidades, creencias, valores y prioridades. Por ello, las sesiones en esta etapa de la enfermedad son una oportunidad para que la familia pueda compartir su aprendizaje, replantearse sus objetivos o expectativas y conformar una rutina que reconozca las limitaciones y potencialidades derivadas de la experiencia oncológica.

Centrándonos en la metodología de intervención, los resultados de investigación extraídos del programa de arteterapia muestran que realizar un acompañamiento terapéutico basado en la acción y la expresión no verbal favorece un sistema de comunicación alternativo y útil para la familia. Habitualmente a los adultos nos resulta complicado acceder a las ideas confusas y a los miedos de los niños respecto al cáncer. La expresión plástica resulta un recurso conocido y atractivo para los niños afectados y sus hermanos, que encuentran en los materiales una herramienta con la que poder mostrar su mundo interno al mismo tiempo que juegan y experimentan. Por ese motivo, una de las funciones del arteterapeuta familiar consiste en orientar a 
los padres y madres para poder escuchar/observar las creaciones plásticas producidas en las sesiones, ayudándoles comprender a sus hijos a través del lenguaje artístico.

Otro de los beneficios observados en el programa de arteterapia es que el proceso creativo compartido por la familia provoca una interacción constante a través de la vivencia práctica. Durante las sesiones los miembros se proponen retos, se piden ayuda, debaten, se aconsejan, comparten frustraciones y logros... La acción creativa promueve un proceso de exploración individual y familiar en la que los miembros se perciben como personas resolutivas y capaces de llevar a cabo proyectos comunes. Por lo tanto, el proceso creativo en sí se convierte en un recurso de observación y de intervención para el arteterapeuta, ayudando a que la familia experimente sistemas relacionales alternativos con los que poder reorganizarse de una forma adaptativa en esta fase de vuelta a casa.

Por último se ha constatado que el lenguaje simbólico es útil a la hora de elaborar aspectos emocionales del cáncer infantil, no solo para los niños afectados, sino también para sus hermanos, padres y madres. A pesar de que en las primeras sesiones a los adultos les cuesta crear e improvisar, a lo largo del programa sus hijos les enseñan a jugar con los materiales sin preocuparse tanto por el resultado estético. Este proceso de acercamiento a la expresión plástica ayuda a desarrollar un discurso personal con el que poder transmitir emociones dolorosas, confusas o complejas a través de elementos gráficos más tolerables para el autor.

El trabajo arteterapéutico con una orientación sistémica permite acompañar a todo el núcleo familiar, respetando los mecanismos de defensa al ofrecer un medio de expresión no invasivo y accesible tanto para niños como para adultos. Los conceptos que cada persona plasma y comparte en sus producciones ayudan a disminuir la represión emocional, el aislamiento o distanciamiento entre los miembros, pudiendo evitar la conformación de algunos tabús habituales en la enfermedad. Del mismo modo, la creación de un discurso metafórico compartido ayuda a los miembros a redefinir su percepción de "familia", fortaleciendo el sentimiento de pertenencia al re-crear unos vínculos capaces de sobreponerse al impacto del cáncer infantil.

\section{Referencias bibliográficas}

1. Grau C, Espada MC. Percepciones de los padres de niños enfermos de cáncer sobre los cambios en las relaciones familiares. Psicooncología 2012;9:125-36. doi: 10.5209/rev PSIC.2012.v9.n1.39142

2. Guijarro A. Estructura y funcionamiento familiar de niños con cáncer desde la perspectiva de las madres (Tesis doctoral). Tenerife: Servicio de publicaciones de la Universidad de la Laguna, 2010.

3. Pitillas, C. La importancia del comportamiento de los padres cuando un hijo tiene cáncer. Madrid: ASION, 2014.

4. Machado F, Jacob E, Castanheira L. Impact of childhood cancer on parent's relationships: An integrative review. J Nurs Scholarship 2010; 42: 250-61. doi: 10.1111/j.15475069.210.01360

5. Espada MC, Grau C. Estrategias de afrontamiento en padres de niños con cáncer. Psicooncología 2012;9: 25-40. doi: 10.5209/rev_PSIC.2012.v9.n1.39136 
6. Cañete A. Fournier C, Bernabeu J, García-Cuenca E, Moran M, Plasencia M, et al. Atención interdisciplinar a las secuelas de la enfermedad y/o tratamientos en oncología pediátrica. Psicooncología 2009;6:381-411.

7. Muniáin AR. El niño con cáncer. Problemática social. Osasunaz, Cuaderno de Ciencias de la Salud 2003; 5: 101-20.

8. Grau C. Impacto psicosocial del cáncer infantil en la familia. Educación, Desarrollo y Diversidad. 2002; 5: 67-87.

9. Lizasoáin O. Educando al niño enfermo. Perspectivas de la pedagogía hospitalaria. Pamplona: Ediciones Eunate, 2000.

10. Celma JA. Participación de la familia en el cuidado del niño oncológico. En: Celma, JA, editor. Psico-oncología pediátrica: valoración e intervención. Sant Vicenç de Torelló: Federación Española de Padres de Niños con Cáncer; 2007, p. 27-30.

11. Llorens À, Mirapeix R, López-Fando T. Hermanos de niños con cáncer: Resultado de una intervención psicoterapéutica. Psicooncología 2009; 6: 469-83.

12. López-Ibor B. Aspectos médicos, psicológicos y sociales del cáncer infantil. Psicooncología 2009;6:281-4.

13. Pedreira JL. El niño enfermo crónico. Aspectos psicosomáticos y relacionales. Psiquis 1998;19:19-26.

14. Dominguez M. (Ed. de Enesco, I.). El cáncer desde la mirada del niño. Madrid: Alianza Editorial, 2009.

15. Grau, C. La atención educativa integral del niño enfermo de cáncer en la escuela inclusiva. Comunicación presentada en: III Jornadas Internacionales de atención integral al niño con cáncer. Sevilla, 1999; p.60-68.

16. Lanzarote MD, Torrado E. Orientación y psicoterapia familiar con niños y adolescentes con enfermedad crónica. Apuntes de Psicología 2009;27: 457-71.

17. Collete, N. Arteterapia y Cáncer. Psicooncología 2011; 11:81-99. doi: 10.5209/rev_ PSIC.2011.v8.n1.7

18. Rico L. Arteterapia en hospitales pediátricos. En: Martinez N, López Fdez.-Cao, editoras. Reinventar la vida. El arte como terapia. Madrid: Eneida; 2009, p. 25-38.

19. Klein J.P. La creación como proceso de transformación. Arteterapia: Papeles de arteterapia y educación artística para la inclusión social. 2006; 1: 11-8.

20. Bengoechea C., Moreira C. Un camino de posibilidades. Experiencias artísticas en una unidad de oncología pediátrica. Arteterapia: Papeles de arteterapia y educación artística para la inclusión social 2013;8: 169-90. doi: 10.5209/rev_ARTE.2013.v8.44444

21. López Fernández Cao M. (Coord.). Creación y posibilidad. Aplicaciones del arte en la integración social. Madrid: Fundamentos, 2006.

22. Alba J. Cama a cama con los más pequeños. En: Martinez N, López Fdez.-Cao, editoras. Reinventar la vida. El arte como terapia. Madrid: Eneida; 2009, p. 39-52.

23. Rico L. Dolor, niños y arte. Oncología y trasplantes pediátricos. Arteterapia: Papeles de arteterapia y educación artística para la inclusión social. 2007; 2: 87-107.

24. Esquembre C, Pérez MS. Arteterapia y oncología pediátrica: Imágenes de la incertidumbre, una manera de convivir con la enfermedad. Comunicación presentada en en: Congreso Internacional de Intervención psicosocial, arte social y arteterapia. De la creatividad al vínculo social. Murcia: Universidad de Murcia, Murcia, 2012.

25. Kerr C. (Coord). Family Art Therapy. Foundations of Theory and Practice. New York: Taylor \& Francis Group, 2008. 
26. Linesch D. Art therapy with families in crisis: Overcoming resistance through nonverbal expression. New York: Brunner/Mazel, 1993.

27. Arrington D. Home is where the art is: An art therapy approach to family therapy. Springfield, Illinois: Charles C. Thomas Publisher, 2001.

28. Shirley R, Malchiodi C. Integrative approaches to family art therapy. Chicago: Magnolia Street Pub, 1994.

29. Gilroy A. Art therapy, research and evidence-based practice. London: SAGE, 2012.

30. Straus A, Corbin J. Bases de la investigación cualitativa: técnicas y procedimientos para desarrollar la teoría fundamentada. Medellín: Universidad de Antioquía, 2002.

31. Gutierrez E. Arteterapia con Familias de Niños con Cáncer. Dinámica Familiar en la creación plástica. (Tesis doctoral). Leioa, Vizcaya: Universidad del País Vasco/Euskal Herriko Unibertsitatea, 2017. 